\title{
Evaluación de la digestión y co-digestión anaerobia de residuos de comida y de poda en bioreactores a escala laboratorio
}

\author{
Evaluation of anaerobic digestion and co-digestion of food \\ waste and grass cuttings in laboratory scale bioreactors
}

\section{Avaliação de digestão e co-digestão anaeróbica de resíduos de alimentos e poda em biorreatores de escala de laboratório}

\author{
Juan Camilo Solarte Toro; Juan Pablo Mariscal Moreno; Beatriz Helena Aristizábal Zuluaga* \\ Departamento de Ingeniería Química. Universidad Nacional de Colombia sede Manizales \\ km 7 vía Aeropuerto La Nubia - Campus la Nubia, Bloque L piso 1, Manizales - Colombia. \\ *bharistizabalz@unal.edu.co
}

Fecha Recepción: 19 de septiembre de 2016 Fecha Aceptación: 2 de mayo de 2017

\begin{abstract}
Resumen
El deterioro ambiental provocado por el alto consumo de combustibles fósiles y la inadecuada disposición de residuos sólidos, ha incentivado su conversión en productos energéticos de alto valor agregado a partir de diferentes procesos biotecnológicos con el fin de compensar parte de la demanda energética. En este trabajo se evalúa la eficiencia, el rendimiento y la productividad del proceso de digestión y co-digestión anaerobia de residuos de comida y poda para valorar su viabilidad como sustratos en la generación de biogás. El seguimiento y evaluación del proceso de digestión mesófila se realizó usando biorreactores por lotes con un volumen activo de 3L, una Relación Inóculo a Sustrato (RIS) de 0,25 (sólidos totales) y una relación residuos de comida a poda de 1,6 (sólidos totales) en el ensayo de co-digestión. En los ensayos realizados se obtuvieron eficiencias de producción de biogás del $38 \%$ y $49 \%$ con respecto al potencial teórico (determinado a partir de la ecuación de Buswell) para los residuos de poda y comida respectivamente en mono-digestión, y un $66 \%$ para la mezcla poda-comida. El modelo de Gompertz de crecimiento de biomasa fue utilizado para simular la generación de biogás en cada proceso. Estos resultados y el seguimiento del $\mathrm{pH}$ durante la digestión, permiten concluir que la mezcla de residuos de comida con residuos de poda mejora los rendimientos de producción de biogás y la estabilidad del sistema en términos de $\mathrm{pH}$, siendo una alternativa para la generación de bioenergía a partir de residuos de cotidiana generación en las ciudades colombianas.
\end{abstract}

Palabras clave: biogás, co-digestión, digestión anaerobia, residuos de comida, residuos de poda.

\begin{abstract}
Environmental degradation caused by high consumption of fossil fuels and improper disposal of solid waste have encouraged its conversion into high value-added products like bioenergy from different biotechnological processes in order to offset some of the energy requirements. This work aims to evaluate the efficiency, performance and productivity of anaerobic digestion and co-digestion processes of food waste and grass cuttings to assess its viability as substrates in biogas generation. The mesophilic anaerobic digestion process was monitored and conducted for selected waste fractions by using $3 \mathrm{~L}$ batch bioreactors (active volume), Inoculum to Substrate Ratio (ISR) of 0.25 (total solids), and food to grass cuttings ratio of 1.6 (total solids). The achieved biogas production efficiencies were $38 \%$ and $49 \%$ for mono-digestion of both grass cuttings and food waste. Biogas generation by co-digestion increased process efficiency up to $66 \%$. The theoretical methane potential was calculated based on Buswell equation
\end{abstract}

Cita: Solarte JC, Mariscal JP, Aristizábal BH. Evaluación de la digestión y co-digestión anaerobia de residuos de comida y de poda en bioreactores a escala laboratorio. rev.ion.2017;30(1):105-116. 
and the Gompertz model for biomass growth was used to simulate the biogas accumulation. The results suggest that mixing food waste to grass cuttings increase biogas yield and prevents an excessive $\mathrm{pH}$ decrease. Co-digestion of these substrates is a good alternative for bioenergy production using daily generated waste in Colombian cities with a higher yield than mono-substrate degradation.

Keywords: biogas, co-digestion, anaerobic digestion, food waste, grass cuttings.

\section{Resumo}

A degradação ambiental, causada pelo alto consumo de combustíveis fósseis e a disposição inadequada dos resíduos sólidos, gerou a transformação desses resíduos em produtos energéticos de alto valor agregado a partir de vários processos biotecnológicos a fim de restaurar parte da demanda de energia. $\mathrm{O}$ objetivo deste trabalho é avaliar a eficiência, o desempenho e a produtividade do processo digestão e codigestão de resíduos de alimentos e poda a fim de estimar a sua viabilidade como substratos na geração de biogás. O acompanhamento e a avaliação do processo de digestão, em condições mesófilas, foram realizados a traves do ensaio do Potencial Químico do Metano (BMP) dos resíduos em biorreatores descontínuos de 3L, com uma Relação Inoculo Substrato (RIS) de 0,25 (sólidos totais) e uma relação resíduo de alimento e poda de 1,6 (sólidos totais) no processo de co-digestão. Nos processos realizados foram obtidas eficiências de produção de metano de 38\%, 49\% e 66\% com respeito ao potencial teórico calculado a partir da equação de Buswell para os resíduos de poda, de comida e a mistura desses respectivamente. O modelo de Gompertz para crescimento de biomassa foi utilizado para simular a acumulação de biogás. Os resultados permitem concluir que a co-digestão de resíduos de alimentos e poda e uma alternativa tangível para gerar bioenergia, a partir do aproveitamento de resíduos que são produzidos na cotidianidade nas cidades colombianas, e apresenta um melhor desempenho do que a degradação mono-substrato.

Palabras-chave: biogás, co-digestão, digestão, resíduos de comida, resíduos de poda.

\section{Introducción}

Colombia es un país en vía de desarrollo que busca aumentar su producción de energía limpia a partir de fuentes renovables y mejorar las prácticas de manejo de Residuos Sólidos Urbanos (RSU). En el país se generan 11,6 millones de toneladas de residuos municipales cada año con una fracción promedio de $65 \%$ en componentes orgánicos. Más del $90 \%$ de los RSU diariamente generados son enviados a relleno sanitario $(81 \%)$ y botaderos a cielo abierto (10,3\%) [1], sitios de disposición que para 321 de los 1096 municipios del país, presentan una vida útil menor a 5 años [2]. Este aspecto, genera la necesidad de diversificación de alternativas de manejo para los RSU generados en Colombia. Adicionalmente, la disposición convencional de residuos orgánicos (residuos de poda y comida) no es conveniente desde el punto de vista ambiental dado que contribuyen a la emisión de Gases de Efecto Invernadero (GEI) y Compuestos Orgánicos Volátiles (COV) [3]. Por ejemplo, las emisiones de GEI relacionados con la disposición de residuos sólidos urbanos fue de 6,16MtonCO $\mathrm{C}_{2}$ e en 2012 en Colombia, con una participación porcentual del departamento de
Caldas de 2,5\% (0,154MtonCO $\mathrm{CO}_{2}$ e/año) [4].

Los residuos de poda y de comida han alcanzado un gran interés para ser degradados en procesos biológicos para la producción de biogás [5] y otros productos de valor agregado como ácidos grasos volátiles [6], hidrógeno [7] y nutrientes ( $\mathrm{N}$ y $\mathrm{P}$ principalmente) [8]. En países como Estados Unidos, los residuos de alimentos son el componente principal de la generación municipal, con aproximadamente 43,6 millones de toneladas enviadas a disposición final cada año [9]. En Australia el valor anual corresponde a 2 millones de toneladas que podrían aprovecharse para la generación de $1915 \mathrm{GW}$ de electricidad anualmente, equivalente al $3,5 \%$ de la energía renovable generada en la actualidad en ese país [10]. En Colombia, de acuerdo con el Atlas de Potencial Energético de la Biomasa Residual [11] los residuos de poda en las 12 principales ciudades tiene un potencial energético de 318,13TJ/año, pero son incipientes los reportes científicos y las aplicaciones a cualquier escala que hagan uso de RSU para la generación de productos de valor agregado.

Al respecto, la cantidad generada y la composición de RSU reportados en diferentes PGIRS (Planes 
de Gestión Integral de Residuos Sólidos) de ciudades colombianas se presenta en la Tabla 1, mostrando el elevado aporte de compuestos orgánicos que son enviados a zonas de disposición final. De acuerdo con los valores presentados en esta tabla, tanto la generación per cápita como la fracción orgánica de los RSU es muy variable entre las ciudades reportadas, aspecto relacionado con el nivel de ingreso de la población, clima, entre otros [12].

Tabla 1. Características de la generación de RSU en algunas ciudades de Colombia [1,13-19].

\begin{tabular}{ccccccc}
\hline TIPO DE RESIDUOS & MANIZALES & BOGOTÁ & QUIBDÓ & CALI & MEDELLÍN & BARRANQUILLA \\
\hline $\begin{array}{c}\text { Total enviado a } \\
\text { disposición (ton/mes) }\end{array}$ & 8492 & 189231 & 2323 & 42896 & 46760 & 38864 \\
$\%$ Alimentos & 26 & 60,6 & $\mathrm{NR}$ & 59 & $\mathrm{NR}$ & $\mathrm{NR}$ \\
$\%$ Poda & 4 & 0,9 & $\mathrm{NR}$ & 6,5 & $\mathrm{NR}$ & 1,5 \\
$\begin{array}{c}\text { \% Orgánicos } \\
\text { NR }\end{array}$ & $\mathrm{NR}$ & 86,17 & $\mathrm{NR}$ & 55,39 & 62,5 \\
$\begin{array}{c}\text { Generación per cápita } \\
\text { (kg/hab-día) }\end{array}$ & 0,7 & 0,3 & 0,6 & 0,4 & 0,6 & 0,9 \\
\hline
\end{tabular}

NR: No reportado.

Las características físicas y químicas de los residuos orgánicos son información importante para el diseño y operación de digestores anaerobios, además de influir en el rendimiento y estabilidad del proceso [9]. Uno de los factores relevantes es la biodegradabilidad, que está relacionado con la cantidad de metano (o biogás) generado y con la cantidad de sólidos (totales o volátiles) destruidos en el proceso [9]. El ensayo del Potencial Bioquímico de Metano (BMP) ha sido usado para cuantificar esta variable, a través de métodos de medición de biogás manométricos o volumétricos en ensayos de laboratorio. Esta medición permite evaluar, de forma preliminar, la viabilidad de un sustrato en procesos anaerobios y el rendimiento máximo alcanzable bajo ciertas condiciones de operación. Angelidaki et al. [20] presentaron un protocolo que permite analizar y expresar los resultados del ensayo de forma que sean comparables, partiendo de condiciones de operación estándar (adición de nutrientes, carga orgánica, frecuencia de muestreo, entre otras variables), que ha sido adaptado en diferentes estudios y con diferentes volúmenes de reacción [21-24].

De acuerdo a las características físicas descritas y a la configuración de las condiciones de operación, la digestión anaerobia mono-sustrato puede presentar algunos inconvenientes que afectan la eficiencia de generación de productos de interés. Teóricamente, debido a la elevada relación $\mathrm{C} / \mathrm{N}$, la digestión de residuos de poda es frecuentemente dominada por una fase de acidificación más rápida que la fase de producción de metano, generando acumulación de Ácidos Grasos Volátiles (AGV) y el descenso del $\mathrm{pH}$, efectos que estresan e inhiben la actividad del consorcio metanogénico [25]. Con este tipo de sustrato, también se presentan dificultades de biodegradación, a causa del alto contenido de lignina y hemicelulosa que impiden la solubilización de carbohidratos [26]. En el caso de residuos de comida, un mayor contenido de proteínas genera un efecto contrario por la posible acumulación de amoniaco, inhibiendo el proceso [25]. A pesar de esto, los compuestos nitrogenados pueden proveer capacidad búfer al ser mezclados con compuestos de elevada relación $\mathrm{C} / \mathrm{N}$ en procesos de co-digestión [25].

Para el caso de la ciudad de Manizales, la elevada producción de residuos de poda puede ser aprovechada en esquemas de co-digestión con residuos de comida, con efectos sinérgicos que puedan generar beneficios que mejoren la estabilidad y desempeño del proceso, con mayores rendimientos en la producción de metano. Se pretende en este documento evaluar la complementariedad de estos residuos como co-sustratos para la generación de biogás en reactores por lotes de $3 \mathrm{~L}$, determinando la eficiencia, el rendimiento, la productividad volumétrica y la variación del pH durante el proceso. Se compara la co-digestión con la degradación mono-sustrato, llevando a cabo cada uno de los ensayos bajo la misma carga orgánica (en términos de SV), temperatura y $\mathrm{pH}$ inicial. La determinación teórica del potencial de producción de biogás, es realizada utilizando la relación estequiométrica de Buswell y mediante el modelo de crecimiento de Gompertz, se pretende simular las condiciones cinéticas. Este trabajo responde a la necesidad de 
encontrar fuentes de aprovechamiento de residuos de poda y como aporte al conocimiento regional en temas de co-digestión.

\section{Metodología}

\section{Sustratos e inóculo}

Los residuos de poda y comida fueron recolectados considerando la norma ASTM D5231-92, la cual tiene como objetivo "Definir y reportar la composición de los residuos sólidos municipales a través de la selección y clasificación manual de las muestras de desecho" [27]. El residuo de poda se tomó de los tres campus de la Universidad Nacional de Colombia sede Manizales. Para evitar la formación de hongos, facilitar su almacenamiento y propiciar iguales características a las muestras, los residuos de poda fueron sometidos a un proceso de secado a $60^{\circ} \mathrm{C}$ hasta alcanzar peso constante [28] y reducción del tamaño de partícula del residuo hasta $1 \mathrm{~mm}$, mediante corte y tamizado. Los residuos de alimentos se obtuvieron en un restaurante de la ciudad, fueron homogeneizados mediante trituración y permanecieron almacenados a $3^{\circ} \mathrm{C}$ hasta su uso en cada uno de los ensayos.

En la caracterización fisicoquímica de cada residuo se determinó el contenido de Sólidos Totales (ST) y Sólidos Volátiles (SV) a través de los métodos estándar APHA (American Public Health Association) para el análisis de agua y aguas residuales [29]. El contenido de Carbono $(\mathrm{C})$, Hidrógeno $(\mathrm{H})$, Oxígeno $(\mathrm{O})$, Nitrógeno $(\mathrm{N})$ y Azufre (S) fue determinado por medio de análisis elemental (métodos ASTM D5373-14 [30] y ASTM D4239-14e2 [31]), y en el caso de los residuos de poda, su contenido de celulosa, hemicelulosa y lignina a través del método de fibras de Van Soest [32].

Como inóculo se utilizó un lodo proveniente del reactor anaerobio de flujo ascendente (UASB) ubicado en el relleno sanitario La Esmeralda, en la ciudad de Manizales. Este inóculo, caracterizado por su alto contenido de humedad, no fue sometido a pretratamiento como concentración o floculación, con el objetivo de hacer una aplicación directa. Angelidaki y colaboradores [20] recomiendan realizar la caracterización de inóculo en términos de ST y SV con el fin de conocer el porcentaje de materia volátil presente, la cual está relacionada con la cantidad de biomasa. No se realizó una evaluación de la actividad metanogénica específica del lodo y este fue desgasificado con el fin de evitar la producción de biogás endógeno en los ensayos, incubando los lodos a $37^{\circ} \mathrm{C}$ durante un periodo de 10 días. Durante el proceso de desgasificación, se añadieron 0,5g/L/día de residuos (mezcla equiporcentual de poda y comida) para adaptar el inóculo a los sustratos empleados.

\section{Potencial experimental de biogás y eficiencia del proceso}

La cantidad máxima real de biogás que se puede obtener a partir de un sustrato bajo determinadas condiciones de operación (temperatura, $\mathrm{pH}$, carga orgánica, etc.) se cuantificó experimentalmente en sistemas herméticos con volumen efectivo de $3 \mathrm{~L}$, de acuerdo con las condiciones presentadas por Angelidaki et al. [20]. La temperatura de operación fue de $37^{\circ} \mathrm{C}$ (controlada utilizando un baño térmico), sin agitación ni control de $\mathrm{pH}$, durante 40 días, con un duplicado cada uno. Las cantidades de inóculo, sustrato y volumen total de operación en cada una de las pruebas se presentan en la Tabla 2. Raposo et al. [21], reportan relaciones experimentales inóculo/sustrato (RIS) entre 0,43 y 3 (ST) en procesos por lotes a partir de diversos sustratos. En este estudio de co-digestión anaerobia se empleó una relación comida a poda de 1,6 (ST), además de mantener en todas las pruebas una relación inóculo a sustrato (RIS) de 0,25 (ST), esta relación evita la flotabilidad del residuo de poda.

Tabla 2. Condiciones de operación en los ensayos de potencial experimental de biogás.

\begin{tabular}{ccccc}
\hline \multirow{2}{*}{ ENSAYO } & $\begin{array}{c}\text { CANTIDAD SUSTRATO } \\
{[\mathrm{g}]}\end{array}$ & $\begin{array}{c}\text { VOLUMEN DE LODO } \\
{[\text { L }]}\end{array}$ & $\begin{array}{c}\text { VOLUMEN TOTAL } \\
{[\text { [L] }}\end{array}$ & SV [g] \\
\hline Poda & 100,5 & & & 86,7 \\
Comida & 307,9 & 1 & 3 & 91,9 \\
Co-digestión & 170 & & & 88,6 \\
\hline
\end{tabular}


Para la cuantificación de biogás se utilizó la técnica de desplazamiento de volumen, ampliamente utilizada debido a su bajo costo, facilidad de montaje y manipulación, durabilidad, escaso requerimiento de mantenimiento y posibilidad de conexión a sistemas de adquisición de datos $[33,34]$. Se utilizó una solución de $\mathrm{NaCl}$ al $80 \%$ $\mathrm{p} / \mathrm{p}$ acidificada a $\mathrm{pH} 0,5$ con ácido sulfúrico con el fin de reducir la solubilidad del dióxido de carbono y cuantificar al máximo la generación de biogás. La reducción de $\mathrm{pH}$ se realiza de acuerdo con las recomendaciones de Walker $[33,35]$ quienes reportan un porcentaje de solubilización de $\mathrm{CO}_{2}$ menor al $8 \%$ cuando se usan soluciones saturadas de $\mathrm{NaCl}$ acidificadas a pH menores a 2 . La solución de $\mathrm{NaCl}$ fue reemplazada luego de 8 días de uso y los valores de biogás son reportados a condiciones estándar de presión y temperatura (1atm y $273,15 K)$.

Se utilizó una solución de nutrientes en cada ensayo, basado en el protocolo expuesto por Angelidaki et al. [20], reemplazando la adición de vitaminas y otros nutrientes con extracto de levadura. Las variables de seguimiento durante un periodo de operación de 40 días fueron $\mathrm{pH}$, temperatura y volumen de solución de $\mathrm{NaCl}$ desplazado con periodos de medición de 24h, aproximadamente.

El seguimiento de las condiciones experimentales permite determinar el rendimiento (REM) y la eficiencia del proceso a partir de las Ecuaciones 1 y 2 , respectivamente.

\section{Generación Teórica de Metano (RTM)}

El potencial teórico de producción de biogás y el contenido de metano que puede ser obtenido en un proceso de digestión anaerobia, está limitado por la estequiometría y puede calcularse si la composición elemental de la materia prima es conocida. A su vez, permite conocer la cantidad de materia orgánica biodegradable [36]. Diferentes modelos estequiométricos se han planteado, siendo uno de los más utilizados el presentado por Buswell y Neave [36-39]. La relación que será utilizada en este trabajo se presenta en la Ecuación 3 [39].

$$
\begin{aligned}
& R E M\left(\frac{N m^{3} \text { biogas }}{k g S V}\right)=\frac{\operatorname{Vbiogas}_{\text {GENERADO }}}{S V_{\text {ALIMENTADO }}} \\
& \operatorname{Eficiencia}(\%)=\frac{R E M}{R T M} \times 100 \% \\
& \mathrm{C}_{n} \mathrm{H}_{a} \mathrm{O}_{b} N_{c}+\left(n-\frac{a}{4}-\frac{b}{2}+\frac{3 c}{4}\right) \mathrm{H}_{2} \mathrm{O} \rightarrow\left(\frac{n}{2}+\frac{a}{8}-\frac{b}{4}-\frac{3 c}{8}\right) \mathrm{CH}_{4}+\left(\frac{n}{2}-\frac{a}{8}+\frac{b}{4}+\frac{3 c}{8}\right) \mathrm{CO}_{2}+c \mathrm{CH}_{3}
\end{aligned}
$$

De acuerdo con Li et al. [39], la Generación Teórica de Metano (RTM) está determinada por la Ecuación 4, haciendo uso de los coeficientes estequiométricos $(n, a, b, c)$ ajustados para cada sustrato y para mezcla a partir de los resultados del análisis elemental.

$$
R T M\left(\frac{m l C H_{4}}{g S V}\right)=\frac{22,4 \times 1000 x\left(\frac{n}{2}+\frac{a}{8}-\frac{b}{4}-\frac{3 c}{8}\right)}{m a s a_{\text {Sustrato }}}
$$

Simulación del proceso de generación de biogás

Asumiendo que la producción de biogás es proporcional a la actividad microbiana, se implementa el ajuste del modelo modificado de crecimiento de Gompertz, utilizada en algunas referencias similares $[40,41]$ para la predicción de la producción de biogás ( $\mathrm{Y}, \mathrm{Nm}^{3}$ biogás/kgSV) a partir de los parámetros cinéticos: producción potencial de biogás ( $\mathrm{A}, \mathrm{Nm}^{3}$ biogás/kgSV), la tasa de producción de biogás $\left(\mu_{\mathrm{m}}, \mathrm{Nm}^{3}\right.$ biogás/kgSV/d) y el tiempo de adaptación $(\lambda, d)$, según la Ecuación 5.

$$
Y=A \exp \left\{-\exp \left[\frac{\mu_{m} e}{A}(\lambda-t)+1\right]\right\}
$$

El ajuste fue realizado empleando la herramienta solver de Excel, minimizando el error entre los valores simulados y los valores experimentales. El grado de ajuste fue determinado por medio del factor de correlación al cuadrado $\left(\mathrm{R}^{2}\right)$. 


\section{Resultados y Discusión}

\section{Características fisicoquímicas de los sustratos e inóculo}

En la Tabla 3 se presentan los datos de la caracterización para ambos sustratos y para el inóculo. El contenido de ST indica una mayor humedad de los residuos de comida $(71 \%)$ en comparación con los residuos de poda $(11,3 \%)$, estos últimos con una mayor proporción de SVIST $(92,1 \%)$. Estos valores son similares a los datos reportados por Prabhudessai et al. [42] de 94,64\% y $97,25 \%$ en SV/ST para residuos de comida. Otros autores como Liu et al. [3] reportan relaciones similares de $77,78 \%$ y $87,53 \%$ en SVIST para la fracción de poda y comida, respectivamente, valores que evidencian la biodegradabilidad potencial de estos residuos.

La relación $\mathrm{C} / \mathrm{N}$ de 19 para residuos de poda es muy baja en comparación con otros reportes, lo que muestra la gran variabilidad y diferencia entre especies consideradas. Brown y Li reportan un valor de $\mathrm{C} / \mathrm{N}$ de 55,3 [37] mientras que Cherosky reporta 143 [43] para residuos de jardinería, mostrando la variabilidad en estos residuos, debido a factores como especies vegetales presentes, localización, tipo de suelo, hora de corte, periodo de crecimiento, entre otras [26]. En lo que respecta a los residuos de comida, el valor de $\mathrm{C} / \mathrm{N}$ de 27 es inferior a reportes, tal como el presentado por Zhang et al. de 14 [9]. Esto indica un posible menor contenido de proteína animal y/o una proporción alta de carbohidratos, propios de las costumbres alimenticias de esta región del país. Las relaciones $\mathrm{C} / \mathrm{N}$ para ambos sustratos se encuentran cerca del intervalo óptimo 20 - 30 [25], lo que indica que pueden ser utilizadas en digestión mono-sustrato al igual que la co-digestión propuesta en este trabajo (C/N de 21).

Tabla 3. Caracterización de sustratos utilizados e inóculo.

\begin{tabular}{ccccc}
\hline PARÁMETRO & UNIDAD & PODA & COMIDA & INÓCULO \\
\hline Sólidos totales & \multirow{2}{*}{$\% / p$} & 88,7 & 29 & 2,2 \\
Sólidos volátiles & & 81,7 & 25,3 & 0,5 \\
SV/ST & $\%$ & 92,1 & 87,2 & 22,7 \\
C & & 44,7 & 48,3 & 12,5 \\
H & & 5,9 & 6,8 & 1,9 \\
N & & 2,7 & 2,1 & 1,6 \\
S & & 0,2 & 0,2 & 3,2 \\
O & $\% \mathrm{p} / \mathrm{p}$ (b.s) & 35,9 & 39 & 9,6 \\
Celulosa & & 26,9 & $\mathrm{NA}$ & $\mathrm{NA}$ \\
Hemicelulosa & & 48,6 & $\mathrm{NA}$ & $\mathrm{NA}$ \\
Lignina & & 24,5 & $\mathrm{NA}$ & $\mathrm{NA}$ \\
C/N & $\mathrm{gmol} / \mathrm{gmol}$ & 19 & 27 & 9,1 \\
Densidad & $\mathrm{kg} / \mathrm{m}^{3}$ & $\mathrm{NA}$ & $\mathrm{NA}$ & 990 \\
\hline
\end{tabular}

$\%$ p/p: peso a peso, b.s.: Base seca, NA: No analizado.

Apesar que el inóculo presenta un bajo contenido de sólidos (2,2\% y $0,5 \%$ en ST y SV, respectivamente) lo que aumenta los requerimientos de volumen en el proceso de digestión, sus características coinciden con aplicaciones reportadas en la literatura para la degradación de residuos de poda. Cherosky reporta como inóculo, el efluente líquido de una planta de tratamiento de aguas residuales con un $10 \%$ de ST, $3,5 \%$ de SV y una relación $\mathrm{C} / \mathrm{N}$ de 7 [43], con productividad de $0,1 \mathrm{~m}^{3} \mathrm{CH}_{4} / \mathrm{kgSV}$ a partir de la co-digestión de residuos de poda y comida (mezcla 25\%-75\%).
Potencial teórico de producción de metano

La fórmula empírica de cada uno de los residuos empleados se obtuvo a partir de la composición elemental presentada en la Tabla 3. En el caso de los residuos de poda esta es $\mathrm{C}_{19} \mathrm{H}_{30} \mathrm{O}_{11} \mathrm{~N}$, para los residuos de alimentos es de $\mathrm{C}_{27} \mathrm{H}_{45} \mathrm{O}_{16} \mathrm{~N}$ y para la mezcla de sustratos es $\mathrm{C}_{21} \mathrm{H}_{34} \mathrm{O}_{13} \mathrm{~N}$ sin considerar el contenido de azufre en cada residuo debido a su bajo porcentaje. Curry y Pillay [38] reportan una fórmula para residuos de comida de $\mathrm{C}_{22} \mathrm{H}_{38} \mathrm{O}_{13} \mathrm{~N}$, similar a la encontrada en este trabajo. La diferencia existente entre las fórmulas empíricas 
determinadas para los residuos de comida puede ser atribuida a la constitución original de estos ya que la variabilidad en la composición depende de factores como el nivel de vida de la región, temporada, cultivos predominantes y costumbres alimenticias [12].

La Tabla 4 presenta las cantidades teóricas de biogás generado (Ecuación 4) así como la composición obtenida a partir de la ecuación de Boyle y el análisis elemental para cada tipo de sustrato. Se presenta también el potencial teórico de la mezcla en el proceso de co-digestión. La composición teórica del biogás obtenida en este estudio para los sustratos considerados se encuentra entre $42,6 \%$ y $52,5 \%$. Prochnow et al. [44] y Prabhudessai et al. [42] reportan porcentajes de $52 \%$ y $50 \%$ v de metano respectivamente, obtenidos para residuos de poda y de comida en ensayos experimentales. Para co-digestión de residuos de poda y de comida Liu et al. reportan porcentajes de metano entre 51,7 y 60,8 [3], superiores a los teóricos obtenidos en este trabajo.

Tabla 4. Producción de biogás teórico y experimental.

\begin{tabular}{|c|c|c|c|c|c|c|}
\hline \multirow[t]{2}{*}{ SUSTRATO } & \multicolumn{3}{|c|}{ COMPOSICIÓN TEÓRICA [\%V/V] } & \multicolumn{2}{|c|}{$\begin{array}{c}\text { Rendimiento acumulado de } \\
\text { biogás } \\
{\left[\mathrm{Nm}^{3} / \mathrm{kg} \mathrm{SV}\right]}\end{array}$} & \multirow[t]{2}{*}{ Eficiencia [\%] } \\
\hline & $\mathrm{CH}_{4}$ & $\mathrm{CO}_{2}$ & $\mathrm{NH}_{3}$ & Teórico & Experimental & \\
\hline Poda & 49,9 & 45,1 & 5 & 0,89 & 0,34 & 38,04 \\
\hline Comida & 52,5 & 43,9 & 3,6 & 0,93 & 0,45 & 48,75 \\
\hline Co-digestión & 42,6 & 53,6 & 3,8 & 0,90 & 0,60 & 66,06 \\
\hline
\end{tabular}

De forma teórica, el efecto de la mezcla de sustratos no genera aumento en la generación de biogás ni en la composición de metano, como se muestra en la Tabla 4. Claramente, la digestión de residuos de comida surge como la alternativa más atractiva, con mayores niveles de producción de $\mathrm{CH}_{4}$ y productividad de biogás. Curry y Pillay reportan un rendimiento teórico en la producción de biogás a partir de residuos de comida de $1 \mathrm{~m}^{3}$ biogás/kgSV [38], el cual es similar al reportado en este trabajo de $099 \mathrm{~m}^{3}$ biogás $/ \mathrm{kgSV}$.

Este método para cuantificar el potencial de producción de metano presenta algunas limitaciones ya que no tiene presente el uso del sustrato para el crecimiento celular ni las restricciones de transferencia de masa y calor que toman lugar en el digestor. Sin embargo, la primera limitación puede considerarse despreciable ya que en el proceso de digestión anaerobia solo se consume entre el $5 \%$ y $10 \%$ del sustrato en producción de biomasa [45]. Por esta razón, los valores obtenidos con este método sobredimensionan los valores de rendimiento y eficiencia y es necesario complementar su análisis con determinaciones experimentales.

\section{Potencial experimental de biogás y eficiencia del proceso}

El rendimiento acumulado de biogás alcanzado de forma experimental en la bio-digestión de cada residuo y su mezcla en la co-digestión se muestra en la Figura 1. El volumen total de biogás generado fue de $0,34,0,45$ y $0,6 \mathrm{Nm}^{3}$ biogás/kgSV para la degradación de residuos de poda, residuos de comida y la mezcla comida-poda, respectivamente. Los resultados muestran el efecto de la co-digestión sobre la producción de biogás, con un aumento de $19.31 \%$ y $28,02 \%$ con respecto a la degradación de poda y de comida, respectivamente. De manera aproximada, considerando la concentración teórica de $\mathrm{CH}_{4}$ presentada en la Tabla 4 para cada ensayo $(49,9,52,5$ y $42,6 \%)$, el potencial experimental de $\mathrm{CH}_{4}$ es de $0,17,0,24$ y $0,26 \mathrm{Nm}^{3} \mathrm{CH}_{4} / \mathrm{kgSV}$ para poda, comida y mezcla. Raposo et al. [21] reportan rendimientos entre 0,27 y $0,37 \mathrm{Nm}^{3} \mathrm{CH}_{4} / \mathrm{kgSV}$ para residuos de poda y entre 0,24 y $0,52 \mathrm{Nm}^{3} / \mathrm{kgSV}$ para residuos de comida. Cadavid-Rodríguez y Bolaños-Valencia reportan rendimientos promedio de metano de $0,33 \mathrm{Nm}^{3} \mathrm{CH}_{4} / \mathrm{kgSV}$ para 9 especies de césped identificados en la ciudad de Palmira, Colombia. Estos autores reportan una remoción 
de sólidos volátiles del $44 \%, 45 \%$ de celulosa, $12 \%$ de hemicelulosa y solo $4 \%$ de lignina [46]. Los rendimientos de Prabhudessai et al. [42], Liu et al. [3] y Massé et al. [47], se encuentran entre 0,2 a $0,31 \mathrm{Nm}^{3} \mathrm{CH}_{4} / \mathrm{kgSV}$ para residuos de poda.

En la Figura 1 se observa que el $80 \%$ del biogás total generado es alcanzado a los 13 días para la codigestión, a los 14 para residuos de poda y a 22 días para la degradación de comida. Zheng et al. plantean que este porcentaje es alcanzado en 10 días en ensayos bajo condiciones termófilas, utilizando residuos de comida como sustrato [9].

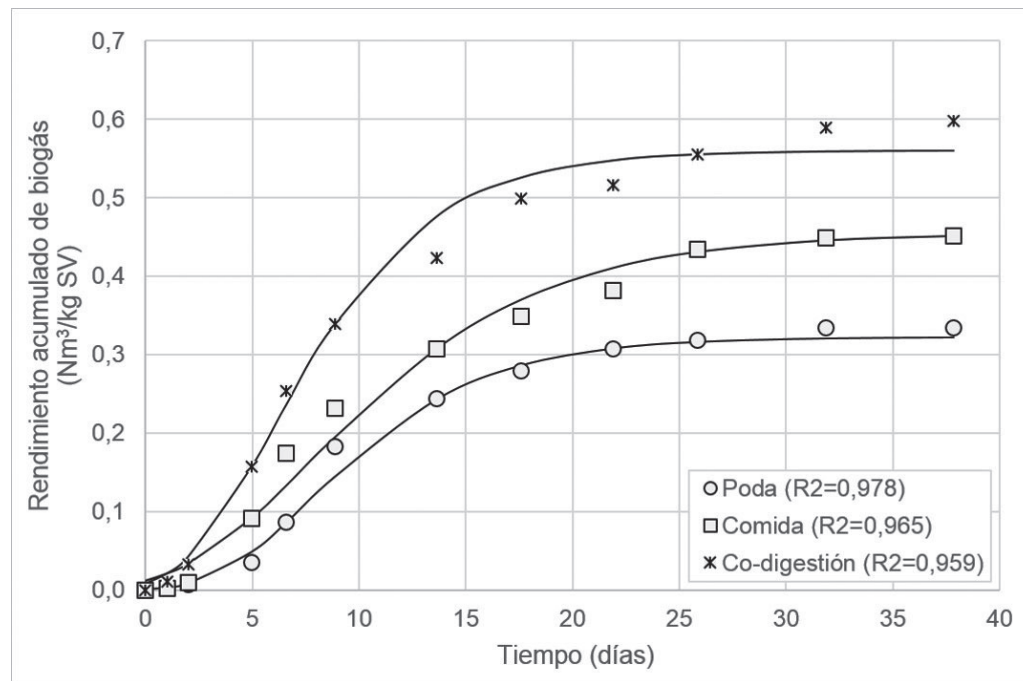

Figura 1. Rendimiento acumulado de biogás para los ensayos realizados.

El ajuste cinético realizado, empleando el modelo de crecimiento de Gompertz modificado (Ecuación 5), permite establecer el periodo de adaptación de cada ensayo, con una buena confiabilidad de acuerdo a los coeficientes de correlación obtenidos $(0,978,0,965$ y 0,959 para la digestión de poda, comida y co-digestión, respectivamente), de acuerdo con la información presentada en la Tabla 5. Los valores de producción potencial de biogás $(A)$ se ajustan adecuadamente a los valores determinados experimentalmente (Tabla 4).
El efecto de la co-digestión se observa en el aumento considerable de la tasa de producción de biogás $\left(\mu_{m}\right)$, pasando de 26,01 y 27,15 para la mono-digestión, a 48,83 $\mathrm{Nm}^{3}$ biogás $/ \mathrm{kgSV} / \mathrm{d}$. La fase de latencia en los 3 ensayos realizados (entre 1,68 y 3,3 días) es baja, en comparación con los reportes de Lo et al. (entre 6,2 y 12,7 días) para la mezcla RSU y cenizas de incinerador [40] pero alta si se compara con lo presentado por Nielfa et al. (entre 0 y 1,5 días) para la co-digestión de RSU con lodos de planta de tratamiento de aguas residuales [41].

Tabla 5. Resultados del modelo de crecimiento de Gompertz para la predicción de la producción de biogás.

\begin{tabular}{ccccc}
\hline \multirow{2}{*}{ SUSTRATO } & $\begin{array}{c}\mathbf{A} \\
{\left[\mathbf{N m}^{3} \text { biogás } / \mathbf{k g ~ S V}\right]}\end{array}$ & $\begin{array}{c}\boldsymbol{\mu}_{\mathrm{m}} \\
{\left[\mathbf{N m}^{3} \text { biogás/kg SV/d] }\right.}\end{array}$ & $\begin{array}{c}\boldsymbol{\lambda} \\
{[\mathbf{d}]}\end{array}$ & $\mathbf{R}^{2}$ \\
\hline Poda & 0,323 & 26,01 & 3,3 & 0,992 \\
Comida & 0,455 & 27,15 & 1,68 & 0,984 \\
Co-digestión & 0,560 & 48,83 & 1,75 & 0,988 \\
\hline
\end{tabular}

\section{Seguimiento a condiciones de operación}

De acuerdo con Sahito et al. [34], junto con el seguimiento a la generación de biogás, la evolución del $\mathrm{pH}$ es un indicador relevante asociado a la actividad del medio de digestión. La evolución del pH en los ensayos considerados se presenta en la Figura 2, en la cual se observa que, con excepción del proceso de mono-digestión de poda, los ensayos mantuvieron los valores de $\mathrm{pH}$ dentro del rango de operación óptimo para este tipo de proceso biológico reportados por Lemos et al. (entre 6,7 a 7,4$)[48]$. 


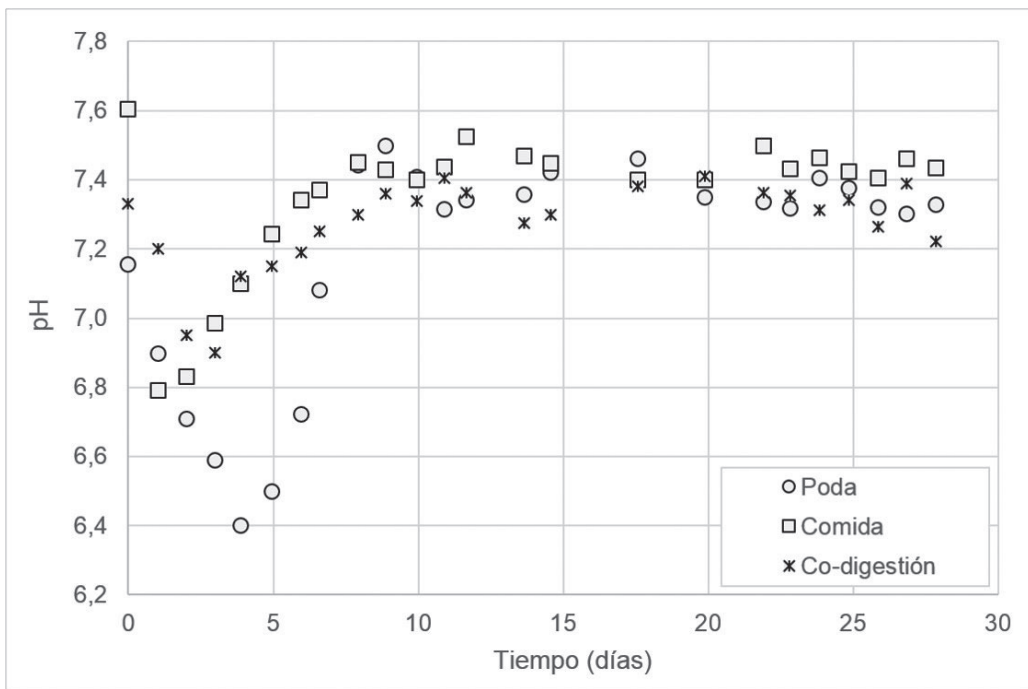

Figura 2. Variación del pH durante la digestión de poda, comida y co-digestión.

En términos generales la variación del $\mathrm{pH}$ durante el proceso de biodigestión presenta una tendencia similar en los tres sustratos utilizados. Se puede observar en la Figura 2 que durante los primeros 6 días se registró una caída del $\mathrm{pH}$ de 0,56 y 0,90 unidades en el caso de los residuos de poda y comida, respectivamente. No obstante, la velocidad en la caída del $\mathrm{pH}$ para estas dos materias primas fue significativamente diferente. Lo anterior se atribuyó a la dificultad que los microorganismos hidrolíticos tienen para romper la matriz lignocelulósica que caracteriza a los residuos de poda tal como se describe en los reportes de Jin et al. [49] y Frigon et al. [50]. Por otro lado, el descenso del $\mathrm{pH}$ en ambos digestores se debió presuntamente a la desigualdad de las velocidades de asimilación y producción de los microorganismos acidogénicos y metanogénicos presentes en cada biodigestor otorgando como resultado una acumulación de Ácidos Grasos Volátiles (AGV) y otros compuestos provenientes de las etapas acidogénica y acetogénica. Sin embargo, dicha disparidad es regulada con el paso del tiempo evitándose así la suspensión del proceso de digestión [51]. En cuanto a la codigestión, se atribuye su estabilidad en el pH al efecto tampón del medio debido a que, durante el proceso, el mayor contenido proteico aportado por los residuos de comida puede proveer una capacidad buffer al mezclarse con residuos de poda [25].

En los ensayos de mono-digestión de residuos de poda reportados por Cadavid et al. [46], los valores más bajos de $\mathrm{pH}$ se obtuvieron en el día 10 del proceso, coincidieron con una mayor concentración de DQO soluble y de AGV. Comparando los ensayos realizados en este trabajo, en términos del comportamiento de $\mathrm{pH}$ mostrado en la Figura 2 , permite establecer que la inclusión de residuos de comida en el proceso de digestión de residuos de poda, mejoran la estabilidad del proceso, reducen la acumulación de AGV y la duración de la fase de hidrólisis.

\section{Conclusiones}

Los resultados experimentales indican que la co-digestión de residuos de poda con residuos de comida incrementó la tasa de generación de biogás, pasando de $0,34 \mathrm{Nm}^{3}$ biogás/kgSV para la mono-digestión de poda, a $0,6 \mathrm{Nm}^{3}$ biogás $/ \mathrm{kgSV}$ para la co-digestión. Estos resultados ofrecen una oportunidad para el aprovechamiento de estos residuos, pero es necesaria la búsqueda de modificaciones para aumentar los niveles de eficiencia en la producción de biogás $(66,1 \%$ para el proceso de co-digestión). Se sugiere el análisis de otros tipos de inóculo y otras relaciones de carga entre sustratos. Por ejemplo, el uso de estiércol como inóculo puede mejorar las condiciones de degradación de compuestos lignocelulósicos.

El conocimiento de la composición elemental de un sustrato es información importante para predecir el potencial de producción de biogás y el nivel de biodegradabilidad. En este documento, el análisis elemental de los residuos utilizados permitió determinar la formula empírica y calcular el rendimiento teórico de producción de metano 
$\left(0,89,0,93,0,9 \mathrm{Nm}^{3}\right.$ biogás $/ \mathrm{kgSV}$ para digestión de residuos de poda, comida y co-digestión, respectivamente). Por otra parte, los modelos de predicción como el de Gompertz, pueden explicar las características cinéticas del proceso de digestión, con correlaciones superiores al $97 \%$. Estas herramientas pueden ahorrar tiempo y costos para la selección de mejores condiciones de operación, con buena confiabilidad.

La mezcla de residuos de comida con residuos de poda, mejora las condiciones operacionales del sistema de digestión. La reducción del pH por debajo de los niveles recomendados $(6,7$ a 7,4$)$ por acumulación de AGV, no se presentó al utilizar residuos de comida como co-sustrato. También se presentó una reducción en los periodos de adaptación, de 3,3 a 1,75 días, valores obtenidos a partir de la simulación del proceso de digestión.

\section{Agradecimientos}

Los autores agradecen a la Universidad Nacional de Colombia sede Manizales el apoyo económico para la realización de este trabajo, en marco del proyecto "Aprovechamiento de residuos sólidos para la generación de energía enfocado a zonas no interconectadas", código Hermes 28452. La profesora Beatriz Helena Aristizábal coordinó parte de este trabajo durante la realización de su año sabático.

\section{Referencias bibliográficas}

[1] Superintendencia de Servicios Públicos Domiciliarios. Disposición Final de Residuos Sólidos - Informe Nacional. 2015.

[2] Departamento Nacional de Planeación. Rellenos sanitarios de 321 municipios colapsarán en cinco años, advierte el DNP. 2016. Disponible en: https://www.dnp.gov. co/Paginas/-Rellenos-sanitarios-de-321municipios-colapsar\%C3\%A1n-en-cincoa\%C3\%B1os,-advierte-el-DNP--.aspx. Fecha de consulta: Julio 2016.

[3] Liu G, Zhang R, El-Mashad H, Dong R. Effect of feed to inoculum ratios on biogas yields of food and green wastes. Bioresour Technol.; 2009;100(21):5103-8.

[4] IDEAM, PNUD, MADS, DNP, Cancillería. Inventario nacional y departamental de gases efecto invernadero - Colombia. Tercera comunicación nacional de cambio climático. Bogotá D.C, 2016.
[5] Chynoweth D, Owens J, Legrand R. Renewable methane from anaerobic digestion of biomass. Renew Energy. 2001;22(1):1-8.

[6] Palomino M, Ortegón M, Rojas T, Martínez J, Valderrama J, Barragán R, et al. Evaluación del potencial acidogénico para producción de AGV de melaza de la industria azucarera como valorización de este subproducto. Rev. ION. 2016;29(1):71-80.

[7] Sreela-or C, Plangklang P, Imai T, Reungsang A. Co-digestion of food waste and sludge for hydrogen production by anaerobic mixed cultures: Statistical key factors optimization. Int. J. Hydrogen Energy. 2011;36(21):1422737.

[8] Romero-Güiza M, Mata-Alvarez J, Chimenos $\mathrm{J}$, Astals S. Nutrient recovery technologies for anaerobic digestion systems: An overview. rev.ion. 2016;29(1):7-26.

[9] Zhang R, El-Mashad H, Hartman K, Wang F, Liu G, Choate C, et al. Characterization of food waste as feedstock for anaerobic digestion. Bioresour Technol. 2007;98(4):929-35.

[10]Lou X, Nair J, Ho G. Potential for energy generation from anaerobic digestion of food waste in Australia. Waste Manag Res. 2013;31(3):283-94.

[11] Unidad de Planeación Minero Energética, Instituto de Hidrología Meteorología y Estudios Ambientales, Colciencias, Universidad Industrial de Santander. Atlas del Potencial Energético de la Biomasa Residual en Colombia. 2011.

[12] Beigl P, Lebersorger S, Salhofer S. Modelling municipal solid waste generation: A review. Waste Manag. 2008;28:200-14.

[13] Alcaldia de Manizales. Plan de gestión integral de residuos sólidos de Manizales PGIRS 2015 - 2027. 2015.

[14]UAESP. Caracterización de los residuos sólidos residenciales en la ciudad de Bogotá D.C 2011. 2011.

[15] Alcaldía Municipal de Quibdó. Actualización y ajustes del plan de gestión integral de residuos sólidos del municipio de Quibdó - Chocó. 2015 - 2026. 2015.

[16] Alcaldia de Santiago de Cali. Evaluación y actualización del plan de gestión integral de residuos sólidos de Santiago De Cali. 2015.

[17] Secretaria de Medio Ambiente. Municipio de Medellín. Actualización del Plan de Gestión Integral de Residuos Sólidos (PGIRS) del municipio De Medellín dando cumplimiento a la Resolución 0754 del 25 de Noviembre del 2014. 2015. 
[18]Alcaldía de Barranquilla. Plan de Gestión Integral de Residuos Sólidos - PGIRS. 2016 - 2027. 2015.

[19] Aristizábal B, Vanegas E, Mariscal J, CamargoValero M. Digestión anaerobia de residuos de poda como alternativa para disminuir emisiones de gases de efecto invernadero en rellenos sanitarios. Revista Energética. 2015;(46):29-36.

[20] Angelidaki I, Alves M, Bolzonella D, Borzacconi L, Campos J, Guwy J, et al. Defining the biomethane potential (BMP) of solid organic wastes and energy crops: a proposed protocol for batch assays. Water Sci Technol. 2009;59(5):927-34.

[21] Raposo F, De la Rubia M, Fernández-Cegrí V, Borja R. Anaerobic digestion of solid organic substrates in batch mode: An overview relating to methane yields and experimental procedures. Renew Sustain Energy Rev; 2012;16(1):861-77.

[22] Scaglia B, Confalonieri R, D'Imporzano G, Adani F. Estimating biogas production of biologically treated municipal solid waste. Bioresour Technol; 2010;101(3):945-52.

[23]Raposo F, Banks C, Siegert I, Heaven S, Borja R. Influence of inoculum to substrate ratio on the biochemical methane potential of maize in batch tests. Process Biochem. 2006;41(6):1444-50.

[24]Davidsson Å, Gruvberger C, Christensen T, Hansen T, Jansen J. Methane yield in sourcesorted organic fraction of municipal solid waste. Waste Manag. 2007;27:406-14.

[25] Esposito G, Frunzo L, Giordano A., Liotta F, Panico A, Pirozzi F. Anaerobic co-digestion of organic wastes. Rev Environ Sci Bio/ Technology. 2012;11(4):325-41.

[26] Nizami A, Orozco A, Groom E, Dieterich B, Murphy J. How much gas can we get from grass? Appl. Energy. 2012;92:783-90.

[27]ASTM International. ASTM D5231-92. Método de prueba estándar para la determinación de la composición de procesado de residuos sólidos urbanos. 2008.

[28] Xie S, Frost J, Lawlor P, Wu G, Zhan X. Effects of thermo-chemical pre-treatment of grass silage on methane production by anaerobic digestion. Bioresour Technol. 2011;102(19):8748-55.

[29] American Public Health Association, American Water Works Association, Water Environment Federation. Standard methods for the examination of water and wastewater. 22nd ed. 2012.

[30]ASTM International. ASTM D5373-14.
Standard Test Methods for Determination of Carbon, Hydrogen and Nitrogen in Analysis Samples of Coal and Carbon in Analysis Samples of Coal and Coke. 2014.

[31]ASTM International. ASTM D4239-14e2. Standard Test Method for Sulfur in the Analysis Sample of Coal and Coke Using High-Temperature Tube Furnace Combustion. 2014.

[32] Van Soest P, Robertson J, Lewis B. Methods for dietary fiber, neutral detergent fiber, and nonstarch polysaccharides in relation to animal nutrition. J. Dairy Sci. 1991;74:3583-97.

[33] Walker M, Zhang $Y$, Heaven S, Banks C. Potential errors in the quantitative evaluation of biogas production in anaerobic digestion processes. Bioresour Technol; 2009;100(24):6339-46.

[34] Sahito A, Mahar R, Rajput M. Development of Volumetric Methane Measurement Instrument for Laboratory Scale Anaerobic Reactors. Mehran Univ Res J Eng Technol. 2015;34(3):309-16.

[35] Carvalho P, Pereira L, Gonçalves N, Queimada A, Coutinho J. Carbon dioxide solubility in aqueous solutions of $\mathrm{NaCl}$ : Measurements and modeling with electrolyte equations of state. Fluid Phase Equilib. 2015;388:100-6.

[36] Chernicharo CA de L. Anaerobic Reactors. London: IWA Publishing; 2007.

[37]Brown D, Li Y. Solid state anaerobic codigestion of yard waste and food waste for biogas production. Bioresour Technol; 2013;127:275-80.

[38] Curry N, Pillay P. Biogas prediction and design of a food waste to energy system for the urban environment. Renew Energy; 2012;41:200-9.

[39] Li Y, Zhang R, Chen C, Liu G, He Y, Liu X. Biogas production from co-digestion of corn stover and chicken manure under anaerobic wet, hemi-solid, and solid state conditions. Bioresour Technol. 2013;149:406-12.

[40] Lo $H$, Kurniawan $T$, Sillanpää $M$, et al. Modeling biogas production from organic fraction of MSW co-digested with MSWI ashes in anaerobic bioreactors. Bioresour. Technol. 2010;101(16):6329-35.

[41] Nielfa A, Cano R, Fdz-Polanco M. Theoretical methane production generated by the codigestion of organic fraction municipal solid waste and biological sludge. Biotechnol Reports. 2015;5:14-21.

[42] Prabhudessai V, Ganguly A, Mutnuri S. Biochemical Methane Potential of Agro Wastes. J Energy. 2013;2013:1-7. 
[43] Cherosky P. Anaerobic Digestion of Yard Waste and Biogas Purification by Removal of Hydrogen Sulfide. The Ohio State University (Tesis de Maestría). 2012.

[44] Prochnow A, Heiermann M, Drenckhan A, Schelle H. Seasonal Pattern of Biomethanisation of Grass from Landscape Management. Agric Eng Int CIGR J; 2005.

[45] Korres N, O'Kiely J, Benzie A, West J. Bioenergy Production by Anaerobic Digestion: Using Agricultural Biomass and Organic Wastes. United Kingdom: Routledge; 2013.

[46] Cadavid-Rodríguez L, Bolaños-Valencia I. Grass from public green spaces an alternative source of renewable energy in tropical countries. rev.ion. 2016;29(1):109-16.

[47] Massé D, Gilbert Y, Savoie P, et al. Methane yield from switchgrass harvested at different stages of development in Eastern Canada.
Bioresour Technol. 2010;101(24):9536-41.

[48] Schmidt A, Borges J, Fonseca W, Monteiro A. Current limitations and the necessary improvements in the anaerobic technology for domestic wastewater treatment. 13th World Congr. Anaerob. Dig. 2013

[49] Jin G, Bierma T, Walker P. Biogas production from switchgrass under experimental conditions simulating US digester operations. J. Environ. Sci. Heal. Part a-Toxic/Hazardous Subst. Environ. Eng. 2012:47(3):470-8.

[50]Frigon J, Mehta P, Guiot S. Impact of mechanical, chemical and enzymatic pretreatments on the methane yield from the anaerobic digestion of switchgrass. Biomass and Bioenergy. 2012:36;1-11.

[51] Abubakar B, Ismail N. Anaerobic digestion of cow dung for biogas production. J. Eng. Appl. Sci. 2012:7(2);169-72. 